Delft University of Technology

\title{
Region-Based Self-Triggered Control for Perturbed and Uncertain Nonlinear Systems
}

Delimpaltadakis, Giannis; Mazo, Manuel

DOI

10.1109/TCNS.2021.3050121

Publication date

2021

Document Version

Final published version

Published in

IEEE Transactions on Control of Network Systems

\section{Citation (APA)}

Delimpaltadakis, G., \& Mazo, M. (2021). Region-Based Self-Triggered Control for Perturbed and Uncertain Nonlinear Systems. IEEE Transactions on Control of Network Systems, 8(2), 757-768.

https://doi.org/10.1109/TCNS.2021.3050121

\section{Important note}

To cite this publication, please use the final published version (if applicable).

Please check the document version above.

Other than for strictly personal use, it is not permitted to download, forward or distribute the text or part of it, without the consent of the author(s) and/or copyright holder(s), unless the work is under an open content license such as Creative Commons.

\section{Takedown policy}

Please contact us and provide details if you believe this document breaches copyrights.

We will remove access to the work immediately and investigate your claim. 
Green Open Access added to TU Delft Institutional Repository

'You share, we take care!' - Taverne project

https://www.openaccess.nl/en/you-share-we-take-care

Otherwise as indicated in the copyright section: the publisher is the copyright holder of this work and the author uses the Dutch legislation to make this work public. 


\title{
Region-Based Self-Triggered Control for Perturbed and Uncertain Nonlinear Systems
}

\author{
Giannis Delimpaltadakis (), Student Member, IEEE, and Manuel Mazo, Jr. (), Senior Member, IEEE
}

\begin{abstract}
In this work, we derive a region-based selftriggered control (STC) scheme for nonlinear systems with bounded disturbances and model uncertainties. The proposed STC scheme is able to guarantee different performance specifications (e.g., stability, boundedness, etc.), depending on the event-triggered control (ETC) triggering function that is chosen to be emulated. To deal with disturbances and uncertainties, we employ differential inclusions (DIs). By introducing ETC/STC notions in the context of DIs, we extend well-known results on ETC/STC to perturbed uncertain systems. Given these results, and adapting tools from our previous work, we derive inner approximations of isochronous manifolds of perturbed uncertain ETC systems. These approximations dictate a partition of the state space into regions, each of which is associated with a uniform intersampling time. At each sampling time instant, the controller checks which region the measured state belongs to and correspondingly decides the next sampling instant.
\end{abstract}

Index Terms-Digital control, networked control systems, nonlinear systems, self-triggered control.

\section{INTRODUCTION}

T HESE DAYS, the use of shared networks and digital platforms for control purposes is becoming more ubiquitous. This has shifted the control community's research focus from periodic to aperiodic sampling techniques, which promish to reduce resource utilization (e.g., bandwidth, processing power, etc.). Arguably, event-based control is the aperiodic scheme that has attracted wider attention, with its two sub-branches being event-triggered control (ETC, e.g., [1]-[5]) and self-triggered control (STC, e.g., [4], [6]-[13]). For an introduction to the topic, the reader is referred to [14].

ETC and STC are sample-and-hold implementations of digital control. In ETC, intelligent sensors monitor continuously the system's state, and transmit data only when a state-dependent triggering condition is satisfied. On the other hand, to tackle the necessity of dedicated intelligent hardware, STC has been proposed, in which the controller at each sampling time instant decides the next one based solely on present measurements.

Manuscript received September 21, 2020; revised November 17, 2020; accepted December 20, 2020. Date of publication January 8, 2021; date of current version August 24, 2021. This work was supported by the ERC Starting Grant SENTIENT 755953. Recommended by Associate Editor D. V. Dimarogonas. (Corresponding author: Giannis Delimpaltadakis.)

The authors are with the Delft Center for Systems and Control, Delft University of Technology, 2628 CD Delft, The Netherlands (e-mail: jmdelimpa@gmail.com; m.mazo@tudelft.nl).

Digital Object Identifier 10.1109/TCNS.2021.3050121
The most common way to decide the next sampling time in STC is the emulation approach: predicting conservatively when the triggering condition of a corresponding ETC scheme would be satisfied. In this way, STC provides the same performance guarantees as the underlying ETC scheme, although it generally leads to faster sampling.

Unfortunately, published work regarding STC for perturbed uncertain nonlinear systems remains very scarce. In [4], ETC and STC schemes are designed for input-to-state stable (ISS) systems subject to disturbances, by employing a small-gain approach. To address model uncertainties, the authors consider nonlinear systems in strict-feedback form and propose a control-design procedure that compensates for the uncertain dynamics, in a way such that the previously derived STC scheme would still guarantee stability. In [10], a self-triggered sampler is derived, which guarantees that the system remains in a safe set, by employing Taylor approximations of the Lyapunov function's derivative. Finally, Tiberi and Johansson [11] design ETC and STC that guarantee uniform ultimate boundedness for perturbed uncertain systems, whereas Tolic et al. [12] employ the small-gain approach to design STC that guarantees $\mathcal{L}_{p}$-stability. Alternative approaches relying on a stochastic framework and learning techniques have also been proposed, see, e.g., [15], [16]. In contrast to the robust approaches listed earlier, they can cope with potentially unbounded disturbances, but they relax the performance guarantees to probabilistic assurances.

Here, we extend the region-based STC framework of Delimpaltadakis and Mazo [13] and propose an STC scheme for general nonlinear systems with bounded disturbances/uncertainties, providing deterministic guarantees. This framework is able to emulate a wide range of triggering conditions and corresponding ETC schemes in a unified generic way. Hence, compared to the deterministic approaches listed earlier, which focus on emulating one class of triggering conditions and provide one specific performance specification, it is more versatile, as it can provide different robust performance guarantees (stability, safety, boundedness, etc.), depending on the ETC scheme that is emulated.

Particularly, in [13], a region-based STC scheme for smooth nonlinear systems has been proposed, which provides intersampling times that lower bound the ideal intersampling times of an a priori given ETC scheme. The state space is partitioned into regions $\mathcal{R}_{i}$, each of which is associated with a uniform intersampling time $\tau_{i}$. The regions $\mathcal{R}_{i}$ are sets delimited by inner approximations of isochronous manifolds (sets composed of points in the state space that correspond to the same ETC 
intersampling time). In a real-time implementation, the controller checks which region the measured state belongs to, and correspondingly decides the next sampling time.

Here, we extend the aforementioned framework to systems with disturbances and uncertainties, which greatly facilitates the applicability of region-based STC in practice. To deal with disturbances and uncertainties in a unified way, we abstract perturbed uncertain systems by differential inclusions (DIs). Moreover, we introduce ETC notions, such as the intersampling time, to the DI framework. Within the DI framework, by employing the notion of homogeneous DIs (see [17]), we are able to extend well-known significant results on ETC/STC, namely, the scaling law of intersampling times [7] and the homogenization procedure [18], to perturbed uncertain systems. Based on these renewed results, we construct approximations of isochronous manifolds of perturbed uncertain ETC systems, thus extending region-based STC to perturbed uncertain systems. We showcase our theoretical results via simulations and comparisons with other deterministic approaches, which indicate that the proposed STC scheme shows competitive performance while simultaneously achieving greater generality.

Apart from the above, let us emphasize that the merits of obtaining approximations of isochronous manifolds extend beyond the context of STC design, as it enables discovering relations between regions in the state space of an ETC system and intersampling times. In fact, such approximations have already been used to construct advanced timing models, which capture the sampling behavior of unperturbed homogeneous ETC systems, and are then used for traffic scheduling in networks of ETC loops (see [19]). More generally, as noted in [13], isochronous manifolds are an inherent characteristic of any system with an output. Thus, implications of the theoretical contribution of approximating them, especially under the effect of disturbances and uncertainties, might even exceed the mere context of ETC/STC.

To summarize our contributions, in this work, we implement the following.

1) Construct a framework based on DIs that allows reasoning about perturbed uncertain ETC systems.

2) Extend important results on ETC/STC to perturbed uncertain systems by employing the DI framework.

3) Obtain approximations of isochronous manifolds of perturbed uncertain ETC systems.

4) Design a robust STC scheme for perturbed uncertain nonlinear systems, which simultaneously achieves greater versatility and competitive performance, compared to the existing literature.

\section{Notation ANd Preliminaries}

\section{A. Notation}

We denote points in $\mathbb{R}^{n}$ as $x$ and their Euclidean norm as $|x|$. For vectors, we also use the notation $\left(x_{1}, x_{2}\right)=\left[\begin{array}{ll}x_{1}^{\top} & x_{2}^{\top}\end{array}\right]^{\top}$. Consider a set $\mathcal{I} \subseteq \mathbb{R}^{n}$. Then, $\overline{\mathcal{I}}$ denotes its closure, $\operatorname{int}(\mathcal{I})$ its interior, and $\operatorname{conv}(\mathcal{I})$ its convex hull. Moreover, for any $\lambda \in \mathbb{R}$, we denote: $\lambda \mathcal{I}=\left\{\lambda x \in \mathbb{R}^{n}: x \in \mathcal{I}\right\}$.
Consider a system of ordinary differential equations (ODE)

$$
\dot{\zeta}(t)=f(\zeta(t))
$$

where $\zeta: \mathbb{R} \rightarrow \mathbb{R}^{n}$. We denote by $\zeta\left(t ; t_{0}, \zeta_{0}\right)$ the solution of (1) with initial condition $\zeta_{0}$ and initial time $t_{0}$. When $t_{0}\left(\right.$ or $\left.\zeta_{0}\right)$ is clear from the context, then it is omitted, i.e., we write $\zeta\left(t ; \zeta_{0}\right)$ (or $\zeta(t)$ ).

Consider the DI

$$
\dot{\zeta}(t) \in F(\zeta(t))
$$

where $\zeta: \mathbb{R} \rightarrow \mathbb{R}^{n}$ and $F: \mathbb{R}^{n} \rightrightarrows \mathbb{R}^{n}$ is a set-valued map. In contrast to ODEs, which under mild assumptions obtain unique solutions given an initial condition, DIs generally obtain multiple solutions for each initial condition, which might even not be defined for all time. We denote by $\zeta\left(t ; \zeta_{0}\right)$ any solution of (2) with initial condition $\zeta_{0}$. Moreover, $\mathcal{S}_{F}([0, T] ; \mathcal{I})$ denotes the set of all solutions of (2) with initial conditions in $\mathcal{I} \subseteq \mathbb{R}^{n}$, which are defined on $[0, T]$. Thus, the reachable set from $\mathcal{I} \subseteq \mathbb{R}^{n}$ of (2) at time $T \geq 0$ is defined as

$$
\mathcal{X}_{T}^{F}(\mathcal{I})=\left\{\xi\left(T ; \xi_{0}\right): \xi\left(\cdot ; \xi_{0}\right) \in \mathcal{S}_{F}([0, T] ; \mathcal{I})\right\} .
$$

Likewise, the reachable flowpipe from $\mathcal{I} \subseteq \mathbb{R}^{n}$ of (2) in the interval $\left[\tau_{1}, \tau_{2}\right]$ is $\mathcal{X}_{\left[\tau_{1}, \tau_{2}\right]}^{F}(\mathcal{I})=\bigcup_{t \in\left[\tau_{1}, \tau_{2}\right]} \overline{\mathcal{X}}_{t}^{F}(\mathcal{I})$.

\section{B. Homogeneous Systems and D/s}

Here, we focus on the classical notion of homogeneity, with respect to the standard dilation. For the general definition and more information, the reader is referred to Bernuau et al. [17] and Kawski [20].

Definition II.1. (Homogeneous functions and set-valued maps): Consider a function $f: \mathbb{R}^{n} \rightarrow \mathbb{R}^{m}$ (or a set-valued map $F: \mathbb{R}^{n} \rightrightarrows \mathbb{R}^{m}$ ). We say that $f($ or $F$ ) is homogeneous of degree $\alpha \in \mathbb{R}$, if for all $x \in \mathbb{R}^{n}$ and any $\lambda>0: f(\lambda x)=\lambda^{\alpha+1} f(x)$ (respectively, $F(\lambda x)=\lambda^{\alpha+1} F(x)$ ).

Correspondingly, a system of ODEs (1) or a DI (2) is called homogeneous of degree $\alpha \in \mathbb{R}$ if the corresponding vector field or set-valued map is homogeneous of the same degree. For homogeneous ODEs or DIs, the following scaling property of solutions holds.

Proposition II.2. (Scaling Property [17], [20]): Let the system of ODEs (1) be homogeneous of degree $\alpha \in \mathbb{R}$. Then, for any $\zeta_{0} \in \mathbb{R}^{n}$ and any $\lambda>0$

$$
\zeta\left(t ; \lambda \zeta_{0}\right)=\lambda \zeta\left(\lambda^{\alpha} t ; \zeta_{0}\right)
$$

Now, let DI (2) be homogeneous of degree $\alpha \in \mathbb{R}$. Then, for any $\mathcal{I} \subseteq \mathbb{R}^{n}$ and any $\lambda>0$

$$
\mathcal{X}_{t}^{F}(\lambda \mathcal{I})=\lambda \mathcal{X}_{\lambda^{\alpha} t}^{F}(\mathcal{I})
$$

\section{ETC Systems}

Consider the control system with state feedback

$$
\dot{\zeta}(t)=f(\zeta(t), v(\zeta(t)))
$$

where $\zeta: \mathbb{R} \rightarrow \mathbb{R}^{n}, f: \mathbb{R}^{n} \times \mathbb{R}^{m_{u}} \rightarrow \mathbb{R}^{n}$, and $v: \mathbb{R}^{n} \rightarrow \mathbb{R}^{m_{u}}$ is the control input. In any sample-and-hold scheme, the control 
input is updated on sampling time instants $t_{i}$ and held constant between consecutive sampling times

$$
\dot{\zeta}(t)=f\left(\zeta(t), v\left(\zeta\left(t_{i}\right)\right)\right), \quad t \in\left[t_{i}, t_{i+1}\right) .
$$

If we define the measurement error as the difference between the last measurement and the present state

$$
\varepsilon_{\zeta}(t):=\zeta\left(t_{i}\right)-\zeta(t), \quad t \in\left[t_{i}, t_{i+1}\right)
$$

then the sample-and-hold system can be written as

$$
\dot{\zeta}(t)=f\left(\zeta(t), v\left(\zeta(t)+\varepsilon_{\zeta}(t)\right)\right), \quad t \in\left[t_{i}, t_{i+1}\right) .
$$

Notice that the error $\varepsilon_{\zeta}(t)$ resets to zero at each sampling time. In ETC, the sampling times are determined by

$$
t_{i+1}=t_{i}+\inf \left\{t>0: \phi\left(\zeta\left(t ; x_{i}\right), \varepsilon_{\zeta}(t)\right) \geq 0\right\}
$$

and $t_{0}=0$, where $x_{i} \in \mathbb{R}^{n}$ is the previously sampled state, $\phi(\cdot, \cdot)$ is the triggering function, (7) is the triggering condition, and $t_{i+1}-t_{i}$ is called intersampling time. Each point $x \in \mathbb{R}^{n}$ corresponds to a specific intersampling time, defined as

$$
\tau(x):=\inf \left\{t>0: \phi\left(\zeta(t ; x), \varepsilon_{\zeta}(t)\right) \geq 0\right\} .
$$

Finally, since $\dot{\varepsilon}_{\zeta}(t)=-\dot{\zeta}(t)$, we can write the dynamics of the extended ETC closed loop in a compact form

$$
\begin{aligned}
\dot{\xi}(t) & =\left[\begin{array}{c}
f\left(\zeta(t), v\left(\zeta(t)+\varepsilon_{\zeta}(t)\right)\right) \\
-f\left(\zeta(t), v\left(\zeta(t)+\varepsilon_{\zeta}(t)\right)\right)
\end{array}\right] \\
& =f_{e}(\xi(t)), t \in\left[t_{i}, t_{i+1}\right) \\
\xi\left(t_{i+1}^{+}\right) & =\left[\begin{array}{c}
\zeta\left(t_{i+1}^{-}\right) \\
0
\end{array}\right]
\end{aligned}
$$

where $\xi=\left(\zeta, \varepsilon_{\zeta}\right) \in \mathbb{R}^{2 n}$. At each sampling time $t_{i}$, the state of (9) becomes $\xi_{i}=\left(x_{i}, 0\right)$. Thus, since we are interested in intervals between consecutive sampling times, instead of writing $\phi\left(\xi\left(t ;\left(x_{i}, 0\right)\right)\right)$ (or $\left.\tau\left(\left(x_{i}, 0\right)\right)\right)$, we abusively write $\phi\left(\xi\left(t ; x_{i}\right)\right)$ (or $\tau\left(x_{i}\right)$ ) for convenience. Between two consecutive sampling times, the triggering function starts from a negative value $\phi\left(\xi\left(t_{i} ; x_{i}\right)\right)<0$, and stays negative until $t_{i+1}^{-}$, when it becomes zero. Triggering functions are designed such that the inequality $\phi\left(\xi\left(t ; x_{i}\right)\right) \leq 0$ implies certain performance guarantees (e.g., stability). Thus, sampling times are defined in a way [see (7)] such that $\phi(\xi(t)) \leq 0$ for all $t \geq 0$, which implies that the performance specifications are met at all time.

\section{STC: Emulation Approach}

The emulation approach to STC entails providing conservative estimates of a corresponding ETC scheme's intersampling times, based solely on the present measurement $x_{i}$

$$
\tau^{\downarrow}\left(x_{i}\right) \leq \tau\left(x_{i}\right)
$$

where $\tau^{\downarrow}(\cdot)$ denotes STC intersampling times. This guarantees that the triggering function of the emulated ETC remains negative at all time, i.e., STC provides the same guarantees as the emulated ETC. Thus, STC intersampling times should be no larger than ETC ones, but as large as possible in order to reduce resource utilization. Finally, infinitely fast sampling (Zeno phenomenon) should be avoided, i.e., $\inf _{x} \tau^{\downarrow}(x) \geq \epsilon>0$.

\section{Problem Statement}

In [13], for a system (9), given a triggering function $\phi(\cdot)$ and a finite set of arbitrary user-defined times $\left\{\tau_{1}, \tau_{2}, \ldots, \tau_{q}\right\}$ (where $\tau_{i}<\tau_{i+1}$ ), which serve as STC intersampling times, the state space of the original system (6) is partitioned into regions $\mathcal{R}_{i} \subset \mathbb{R}^{n}$ such that

$$
\forall x \in \mathcal{R}_{i}: \quad \tau_{i} \leq \tau(x)
$$

where $\tau(x)$ denotes ETC intersampling times corresponding to the given triggering function $\phi(\cdot)$. The region-based STC protocol operates as follows.

1) Measure the current state $\xi\left(t_{k}\right)=\left(x_{k}, 0\right)$.

2) Check to which of the regions $\mathcal{R}_{i}$ the point $x_{k}$ belongs.

3) If $x_{k} \in \mathcal{R}_{i}$, set the next sampling time to $t_{k+1}=t_{k}+\tau_{i}$.

As mentioned in Section I, we aim at extending the STC technique of Delimpaltadakis and Mazo [13] to systems with disturbances and uncertainties. Thus, we consider perturbed/uncertain ETC systems, written in the compact form

$$
\dot{\xi}(t)=\left[\begin{array}{c}
f\left(\zeta(t), v\left(\zeta(t)+\varepsilon_{\zeta}(t)\right), d(t)\right) \\
-f\left(\zeta(t), v\left(\zeta(t)+\varepsilon_{\zeta}(t)\right), d(t)\right)
\end{array}\right]=f_{e}(\xi(t), d(t))
$$

where $d: \mathbb{R} \rightarrow \mathbb{R}^{m_{d}}$ is an unknown signal (e.g., disturbance, model uncertainty, etc.), and assume that a triggering function $\phi(\xi(t))$ is given.

Assumption 1: For the remainder of this article, we assume the following.

1) The function $f_{e}(\cdot, \cdot)$ is locally bounded and continuous with respect to all of its arguments.

2) For all $t \geq 0$ : $d(t) \in \Delta$, where $\Delta \subset \mathbb{R}^{m_{d}}$ is convex, compact, and nonempty.

3) The function $\phi(\cdot)$ is continuously differentiable.

4) For all $\xi_{0}=\left(x_{0}, 0\right) \in \mathbb{R}^{2 n}: \phi\left(\xi_{0}\right)<0$. Moreover, for any compact set $K \subset \mathbb{R}^{n}$ there exists $\epsilon_{K}>0$ such that for all $x_{0} \in K$ and any $d(t)$, with $d(t) \in \Delta$ for all $t \geq 0$, $\phi\left(\xi\left(t ; \xi_{0}\right)\right)<0$ for all $t \in\left[0, \epsilon_{K}\right)$.

The problem statement of this work is as follows.

Problem Statement: Given a system (12) and a triggering function $\phi(\cdot)$, which satisfy Assumption 1, and a predefined finite set of times $\left\{\tau_{1} \ldots, \tau_{q}\right\}$ (with $\tau_{i}<\tau_{i+1}$ ), derive regions $\mathcal{R}_{i} \subset \mathbb{R}^{n}$ that satisfy (11).

Remark 1: In region-based STC, the Zeno phenomenon is ruled out by construction, since region-based STC intersampling times are lower bounded: $\tau^{\downarrow}(x) \geq \min _{i} \tau_{i}=\tau_{1}$.

Items 1) and 2) of Assumption 1 impose the satisfaction of the standard assumptions of DIs on the DIs that we construct later [see (17)]. These assumptions ensure existence of solutions for all initial conditions (see [17] and [21] for more details). Note that assuming convexity of $\Delta$ is not restrictive, since in the case of a nonconvex $\Delta$, we can consider the closure of its convex hull and write $d(t) \in \overline{\operatorname{conv}(\Delta)}$ for all $t \geq 0$. Finally, item 3 ) is employed in the proof of Lemma VI.1, whereas item 4) ensures that the emulated ETC associated with the given 
triggering function does not exhibit Zeno behavior for any given bounded state.

Remark 2: The triggering function should be chosen to be robust to disturbances/uncertainties, such that the emulated ETC does not exhibit Zeno behavior. Examples of such robust triggering functions are as follows:

1) Lebesgue sampling (e.g., [1], [11]): $\phi(\xi(t))=$ $\left|\varepsilon_{\zeta}(t)\right|^{2}-\epsilon^{2}$, where $\epsilon>0$

2) mixed-triggering (e.g., [4]): $\mathrm{x} \quad \phi(\xi(t))=\left|\varepsilon_{\zeta}(t)\right|^{2}-$ $\sigma|\zeta(t)|^{2}-\epsilon^{2}$, where $\sigma>0$ is appropriately chosen and $\epsilon>0$.

Both functions satisfy Assumption 1.

\section{Overview of Delimpaltadakis and Mazo [13]}

In this section, we give a brief overview of Delimpaltadakis and Mazo [13]. First, we focus on homogeneous ETC systems and how, exploiting approximations of their isochronous manifolds, a state-space partitioning into regions $\mathcal{R}_{i}$ can be derived. Afterward, we recall how these results can be generalized to general nonlinear systems, by employing a homogenization procedure.

\section{A. Homogeneous ETC Systems, Isochronous Manifolds, and State-Space Partitioning}

An important property of homogeneous ETC systems is the scaling of intersampling times.

Theorem IV.1. (Scaling of ETC intersampling Times [7]): Consider an ETC system (9) and a triggering function, homogeneous of degree $\alpha$ and $\theta$, respectively. Then, for all $x \in \mathbb{R}^{n}$ and $\lambda>0$

$$
\tau(\lambda x)=\lambda^{-\alpha} \tau(x)
$$

where $\tau(\cdot)$ is defined in (8).

Thus, for homogeneous ETC systems with degree $\alpha>0$, along a ray that starts from the origin (homogeneous ray), intersampling times become larger for points closer to the origin. Scaling law (13) is a direct consequence of the system's and triggering function's homogeneity, since (3) implies that

$$
\phi(\xi(t ; \lambda x))=\phi\left(\lambda \xi\left(\lambda^{\alpha} t ; x\right)\right)=\lambda^{\theta+1} \phi\left(\xi\left(\lambda^{\alpha} t ; x\right)\right) .
$$

In [13], the scaling law (13) is combined with inner approximations of isochronous manifolds, a notion first introduced in [18]. Isochronous manifolds are sets of points with the same intersampling time.

Definition IV.2. (Isochronous Manifolds): Consider an ETC system (9). The set $M_{\tau_{\star}}=\left\{x \in \mathbb{R}^{n}: \tau(x)=\tau_{\star}\right\}$, where $\tau(x)$ is defined in (8), is called isochronous manifold of time $\tau_{\star}$.

For homogeneous systems, the scaling law (13) implies that isochronous manifolds satisfy the following properties.

Proposition IV.3. (see [13], [18]): Consider an ETC system (9) and a triggering function, homogeneous of degree $\alpha>0$ and $\theta>0$, respectively, and let Assumption 1 hold. Then, the following hold.

1) For any time $\tau_{\star}>0$, there exists an isochronous manifold $M_{\tau_{\star}}$.
2) Isochronous manifolds are hypersurfaces of dimension $n-1$.

3) Each homogeneous ray intersects an isochronous manifold $M_{\tau_{\star}}$ only at one point.

4) Given two isochronous manifolds $M_{\tau_{1}}$ and $M_{\tau_{2}}$ with $\tau_{1}<\tau_{2}$, on every homogeneous ray, $M_{\tau_{1}}$ is further away from the origin compared to $M_{\tau_{2}}$, i.e., for all $x \in M_{\tau_{1}}$

$$
\begin{aligned}
& \exists ! \lambda_{x} \in(0,1) \text { s.t. } \lambda_{x} x \in M_{\tau_{2}} \\
& \exists \kappa_{x} \geq 1 \text { s.t. } \kappa_{x} x \in M_{\tau_{2}} .
\end{aligned}
$$

Properties 2)-4) from Proposition IV.3 are illustrated in Fig. 1(a). Now, consider the region $R_{1}$ between isochronous manifolds $M_{\tau_{1}}$ and $M_{\tau_{2}}$ in Fig. 1(a). The scaling law (13) directly implies that for all $x \in R_{1}: \tau_{1} \leq \tau(x)$, i.e., (11) is satisfied. Thus, if isochronous manifolds could be computed, then the state space could be partitioned into the regions delimited by isochronous manifolds and the region-based STC scheme would be enabled.

\section{B. Inner Approximations of Isochronous Manifolds}

Since isochronous manifolds cannot be computed analytically, in [13], inner approximations $\underline{M}_{\tau_{i}}$ of isochronous manifolds $M_{\tau_{i}}$ are derived in an analytic form [see Fig. 1(b)]. Again, due to the scaling law, for the region $\mathcal{R}_{1}$ between two inner approximations $\underline{M}_{\tau_{1}}$ and $\underline{M}_{\tau_{2}}$ (with $\tau_{1}<\tau_{2}$ ), it holds that $\tau_{1} \leq \tau(x)$ for all $x \in \mathcal{R}_{1}$. Hence, given a set of times $\left\{\tau_{1}, \ldots, \tau_{q}\right\}$, the state space is partitioned into regions $\mathcal{R}_{i}$ delimited by these inner approximations. As noted in [13], it is crucial that approximations $\underline{M}_{\tau_{i}}$ have to satisfy the same properties as isochronous manifolds, mentioned in Proposition IV.3. For example, if approximations $\underline{M}_{\tau_{i}}$ did not satisfy properties 3 ) and 4 ), then $\mathcal{R}_{i}$ could potentially intersect with each other and be ill-defined [see Fig. 1(c)].

To derive the inner approximations, the triggering function $\phi(\xi(t ; x))$ is upper bounded by a function $\mu(x, t)$ with linear dynamics that satisfies certain conditions. Then, the sets $\underline{M}_{\tau_{i}}=\left\{x \in \mathbb{R}^{n}: \mu\left(x, \tau_{i}\right)=0\right\}$ are proven to be inner approximations of isochronous manifolds $M_{\tau_{i}}$. The sufficient conditions that $\mu(x, t)$ has to satisfy in order for its zero-level sets to be inner approximations of isochronous manifolds and satisfy the properties mentioned in Proposition IV.3 are summarized in the following theorem.

Theorem IV.4. (see [13]): Consider an ETC system (9) and a triggering function $\phi(\cdot)$, homogeneous of degrees $\alpha>0$ and $\theta>0$, respectively. Let $\mu: \mathbb{R}^{n} \times \mathbb{R}^{+} \rightarrow \mathbb{R}$ be a function that satisfies

$$
\begin{aligned}
& \mu(x, 0)<0 \quad \forall x \in \mathbb{R}^{n} \backslash\{0\} \\
& \mu(x, t) \geq \phi(\xi(t ; x)) \quad \forall t \in[0, \tau(x)] \text { and } \quad \forall x \in \mathbb{R}^{n} \backslash\{0\}
\end{aligned}
$$

$$
\mu(\lambda x, t)=\lambda^{\theta+1} \mu\left(x, \lambda^{\alpha} t\right) \quad \forall t, \lambda>0 \text { and } \quad \forall x \in \mathbb{R}^{n} \backslash\{0\}
$$

$\forall x \in \mathbb{R}^{n} \backslash\{0\}: \quad \exists ! \tau_{x}>0$ such that $\mu\left(x, \tau_{x}\right)=0$. 


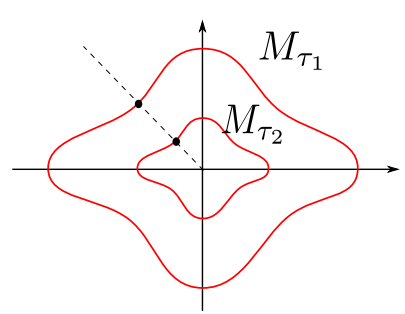

(a)

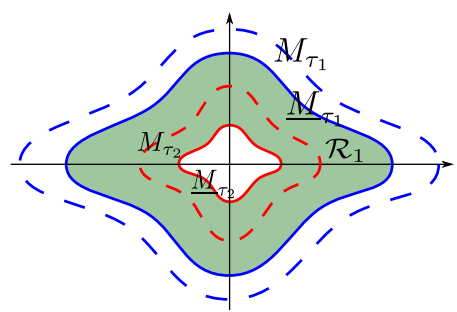

(b)

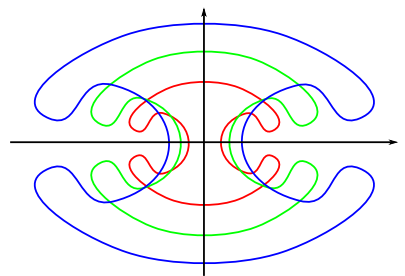

(c)

Fig. 1. Isochronous manifolds and inner approximations. (a) Isochronous manifolds of a homogeneous ETC system for times $\tau_{1}<\tau_{2}$. (b) Isochronous manifolds $M_{\tau_{i}}$ (dashed lines) and inner approximations $\underline{M}_{\tau_{i}}$ (solid lines). The filled region represents $\mathcal{R}_{1}$. (c) If inner approximations of isochronous manifolds did not satisfy properties 3) and 4) from Proposition IV.3, then the regions $\mathcal{R}_{i}$ could intersect with each other.

The sets $\underline{M}_{\tau_{\star}}:=\left\{x \in \mathbb{R}^{n}: \mu\left(x, \tau_{\star}\right)=0\right\}$ are inner approximations of isochronous manifolds $M_{\tau_{\star}}$. Moreover, the sets $\underline{M}_{\tau_{\star}}$ satisfy the properties mentioned in Proposition IV.3.

Let us briefly explain what is the intuition behind this theorem. Since (15a) and (15b) hold, if we denote by $\tau^{\downarrow}(x):=$ $\inf \{t>0: \mu(x, t)=0\}$ [from (15d) we know that it exists], then $\tau^{\downarrow}(x) \leq \tau(x)$. Note that it is important that inequality (15b) extends at least until $t=\tau(x)$, in order for $\tau^{\downarrow}(x) \leq$ $\tau(x)$. Then, by the scaling law (13), we have that the set $\underline{M}_{\tau_{\star}}=\left\{x \in \mathbb{R}^{n}: \tau^{\downarrow}(x)=\tau_{\star}\right\}$ is an inner approximation of the isochronous manifold $M_{\tau_{\star}}$. Moreover, since for each $x$, the equation $\mu(x, t)=0$ has a unique solution w.r.t. $t$ [from (15d)], we get that $\underline{M}_{\tau_{\star}} \equiv\left\{x \in \mathbb{R}^{n}: \mu\left(x, \tau_{\star}\right)=0\right\}$. Finally, condition (15c) implies that $\tau^{\downarrow}(\lambda x)=\lambda^{-\alpha} \tau^{\downarrow}(x)$ [observe the similarity between (15c) and (14)], which, in turn, implies that the sets $\underline{M}_{\tau_{\star}}$ satisfy the properties of Proposition (IV.3). We do not elaborate more on the technical details here (e.g., how is the bounding carried out), since we address these later in the document, where we extend the theoretical results of Delimpaltadakis and Mazo [13] to perturbed/uncertain systems.

\section{Homogenization of Nonlinear Systems and Region-Based STC}

To exploit aforementioned properties of homogeneous systems, the homogenization procedure proposed by Anta and P. Tabuada [18] is employed. Any nonhomogeneous system (9) is rendered homogeneous of degree $\alpha>0$, by embedding it into $\mathbb{R}^{2 n+1}$ and adding a dummy variable $w$

$$
\left[\begin{array}{c}
\dot{\xi} \\
\dot{w}
\end{array}\right]=\left[\begin{array}{c}
w^{\alpha+1} f_{e}\left(w^{-1} \xi\right) \\
0
\end{array}\right]=\tilde{f}_{e}(\xi, w) .
$$

The same can be done for nonhomogeneous triggering functions $\tilde{\phi}(\xi, w)=w^{\theta+1} \phi\left(w^{-1} \xi\right)$. Notice that the trajectories of the original ETC system (9) with initial condition $\left(x_{0}, e_{0}\right) \in \mathbb{R}^{2 n}$ coincide with the trajectories of the homogenized one (16) with initial condition $\left(x_{0}, e_{0}, 1\right) \in \mathbb{R}^{2 n+1}$, projected to the $\xi$ variables. The same holds for a homogenized triggering function. Thus, the intersampling times $\tau\left(x_{0}\right)$ of system (9) with triggering function $\phi(\cdot)$ coincide with the intersampling times $\tau\left(\left(x_{0}, 1\right)\right)$ of (16) with triggering function $\tilde{\phi}(\cdot)$.

Consequently, if the original system (or the triggering function) is nonhomogeneous, then first it is rendered homogeneous

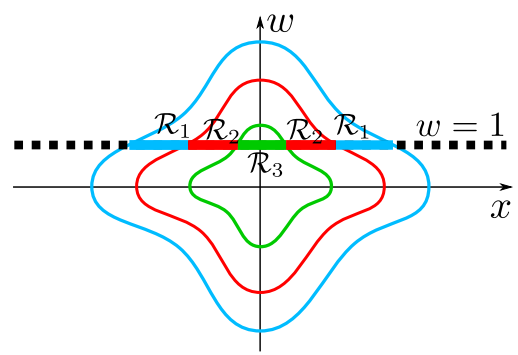

Fig. 2. Inner approximations of isochronous manifolds (colored curves) for a homogenized system (16) and the regions $\mathcal{R}_{i}$ between them. The colored segments on the $w=1$ plane represent the subsets of the hyperplane $w=1$ (i.e., the subsets of the original state space) that are contained in the regions $\mathcal{R}_{i}$ and are associated with the corresponding intersampling times $\tau_{i}$.

via the homogenization procedure (16). Afterward, inner approximations of isochronous manifolds for the homogenized system (16) are derived. Since trajectories of the original system are mapped to trajectories on the $w=1$ plane of the homogenized one (i.e., the state space of the original system is mapped to the $w=1$ plane), to determine the intersampling time $\tau_{i}$ of a state $x_{0} \in \mathbb{R}^{n}$, one has to check to which region $\mathcal{R}_{i} \subset \mathbb{R}^{n+1}$ the point $\left(x_{0}, 1\right)$ belongs. For an illustration, see Fig. 2: e.g., given a state $x_{0} \in \mathbb{R}^{n}$, if $\left(x_{0}, 1\right) \in \mathbb{R}^{n+1}$ lies on the cyan segment (i.e., it is contained in $\mathcal{R}_{1}$ ), then the STC intersampling time that is assigned to $x_{0}$ is $\tau^{\downarrow}\left(x_{0}\right)=\tau_{1}$.

Note that, here, it suffices to inner-approximate the isochronous manifolds of (16) only in the subspace $w>0$, since we only care about determining regions $\mathcal{R}_{i}$ for points $\left(x_{0}, 1\right) \in \mathbb{R}^{2 n}$. Thus, the conditions of Theorem IV.4 can be relaxed so that they hold only in the subspace $w>0$, i.e., for all $(x, w) \in\left(\mathbb{R}^{n} \backslash\{0\}\right) \times \mathbb{R}_{+}$.

\section{Perturbed/Uncertain ETC Systems as DIs}

In this section, we show how a general perturbed/uncertain nonlinear system (12), satisfying Assumption 1, can be abstracted by a homogeneous DI. Moreover, we extend the notion of intersampling times in the context of DIs and show that scaling law (13) holds for intersampling times of homogeneous DIs. These results are used afterward in Section VI to derive inner 
approximations of isochronous manifolds of perturbed/uncertain systems (12), and thus enable the region-based STC scheme.

\section{A. Abstractions by D/s}

Notice that since system (12) is a time-varying system, many notions that we introduced before for time-invariant systems are now ill-defined. For example, depending on the realization of the unknown signal $d(t)$, a sampled state $x \in \mathbb{R}^{n}$ can correspond to different intersampling times, i.e., definition (8) is ill-posed. However, employing item 2) of Assumption 1 and the notion of DIs, we can abstract the behavior of the family of systems (12) and remove such dependencies. In particular, system (12) can be abstracted by the following DI:

$$
\dot{\xi}(t) \in F(\xi(t)):=\left\{f_{e}(\xi(t), d(t)): d(t) \in \Delta\right\} .
$$

For DI (17) [i.e., for the family of systems (12)], the intersampling time $\tau(x)$ of a point $x \in \mathbb{R}^{n}$ can now be defined as the worst-case possible intersampling time of $x$, under any possible signal $d(t)$ satisfying Assumption 1 .

Definition V.1. (Intersampling Times of DI): Consider the family of systems (12), the DI (17) abstracting them, and a triggering function $\phi: \mathbb{R}^{2 n} \rightarrow \mathbb{R}$. Let Assumption 1 hold. For any point $x \in \mathbb{R}^{n}$, we define its intersampling time as

$$
\tau(x):=\inf \left\{t>0: \sup \left\{\phi\left(\mathcal{X}_{t}^{F}((x, 0))\right)\right\} \geq 0\right\} .
$$

Note that we have already emphasized that we consider initial conditions $(x, 0) \in \mathbb{R}^{2 n}$, since at any sampling time, the measurement error $\varepsilon_{\zeta}=0$. Finally, now that intersampling times of systems (12) abstracted by DIs are well-defined, we can accordingly redefine isochronous manifolds for families of such systems as: $M_{\tau_{\star}}=\left\{x \in \mathbb{R}^{n}: \tau(x)=\tau_{\star}\right\}$, where $\tau(x)$ is defined in (18).

\section{B. Homogenization of DIs and Scaling of Intersampling Times}

As previously mentioned, the scaling law of intersampling times (13) for homogeneous systems is of paramount importance for the approach of Delimpaltadakis and Mazo [13]. We show that a similar result can be derived for intersampling times (18) of DIs. First, observe that DI (17) can be rendered homogeneous of degree $\alpha>0$, by slightly adapting the homogenization procedure (16) as follows:

$$
\left[\begin{array}{c}
\dot{\xi}(t) \\
\dot{w}(t)
\end{array}\right] \in \tilde{F}(\xi(t), w(t))
$$

where $\quad \tilde{F}(\xi, w):=\left[\begin{array}{c}\left\{w^{\alpha+1} f_{e}\left(w^{-1} \xi, d(t)\right): d(t) \in \Delta\right\} \\ \{0\}\end{array}\right] . \quad$ Indeed, $\tilde{F}(\cdot, \cdot)$ is homogeneous of degree $\alpha$. Recall that the same can be done for a nonhomogeneous triggering function

$$
\tilde{\phi}(\xi, w)=w^{\theta+1} \phi\left(w^{-1} \xi\right) .
$$

Again, trajectories and flowpipes of (17) with initial condition $\left(x_{0}, e_{0}\right) \in \mathbb{R}^{2 n}$ coincide with the projection to the $\xi$-variables of trajectories of (19) with initial condition $\left(x_{0}, e_{0}, 1\right) \in \mathbb{R}^{2 n+1}$.
This implies that the intersampling time $\tau\left(x_{0}\right)$ for DI (17) with triggering function $\phi(\cdot)$, defined as in (18), is the same as the intersampling time $\tau\left(\left(x_{0}, 1\right)\right)$ for DI (19) with triggering function $\tilde{\phi}(\cdot)$.

Given the above, by employing the scaling property (4) of flowpipes of homogeneous DIs, we can prove that the scaling law holds for intersampling times of DIs (19).

Theorem V.2: Consider DI (19), the triggering function $\tilde{\phi}(\cdot)$ from (20), and let Assumption 1 hold. The intersampling time $\tau((x, w))$, where $(x, w) \in \mathbb{R}^{n+1}$, scales for any $\lambda>0$ as

$$
\tau(\lambda(x, w))=\lambda^{-\alpha} \tau((x, w))
$$

where $\tau(\cdot)$ is defined in (18).

Proof: See the Appendix.

For an example of how DIs and triggering functions are homogenized, the reader is referred to Section VII.

\section{Region-Based StC for Perturbed/ UNCERTAIN SYSTEMS}

In this section, we use the previous derivations about DIs to inner-approximate isochronous manifolds of perturbed/uncertain systems, by adapting the technique of Delimpaltadakis and Mazo [13]. Using the derived inner approximations, the state-space partitioning into regions $\mathcal{R}_{i}$ is generated. Finally, we show that the applicability of region-based STC for perturbed/uncertain systems is semiglobal.

\section{A. Approximations of Isochronous Manifolds of Perturbed/Uncertain ETC Systems}

Similarly to Delimpaltadakis and Mazo [13], we upper-bound the time evolution of the (homogenized) triggering function $\tilde{\phi}(\xi(t ; x), w(t))$ along the trajectories of DI (19) with a function $\mu((x, w), t)$ in analytic form that satisfies (15). For this purpose, first we provide a lemma, similar to the comparison lemma [22] and to [13, Lemma V.2], which shows how to derive upper bounds with linear dynamics of functions evolving along flowpipes of DIs.

Lemma VI.1: Consider a system of ODEs

$$
\dot{\xi}(t)=f(\xi(t), d(t))
$$

where $\xi(t) \in \mathbb{R}^{n}, d(t) \in \mathbb{R}^{m_{d}}, f: \mathbb{R}^{n} \times \mathbb{R}^{m_{d}} \rightarrow \mathbb{R}^{n}$, and the function $\phi: \mathbb{R}^{n} \rightarrow \mathbb{R}$. Let $f, d$, and $\phi$ satisfy Assumption 1 . Consider the DI abstracting the family of ODEs (22)

$$
\dot{\xi}(t) \in F(\xi(t)):=\{f(\xi(t), d(t)): d(t) \in \Delta\} .
$$

Consider a compact set $\Xi \subseteq \mathbb{R}^{n}$. For coefficients $\delta_{0}, \delta_{1} \in \mathbb{R}$ satisfying

$$
\frac{\partial \phi}{\partial z}(z) f(z, u) \leq \delta_{0} \phi(z)+\delta_{1} \quad \forall z \in \Xi \text { and } \quad \forall u \in \Delta
$$

the following inequality holds for all $\xi_{0} \in \Xi$ :

$$
\sup \left\{\phi\left(\mathcal{X}_{t}^{F}\left(\xi_{0}\right)\right)\right\} \leq \psi\left(y\left(\xi_{0}\right), t\right) \quad \forall t \in\left[0, t_{e}\left(\xi_{0}\right)\right]
$$

where $t_{e}\left(\xi_{0}\right)$ is defined as the escape time

$$
t_{e}\left(\xi_{0}\right)=\inf \left\{t>0: \mathcal{X}_{t}^{F}\left(\xi_{0}\right) \nsubseteq \Xi \Xi\right\}
$$


and $\psi\left(y\left(\xi_{0}\right), t\right)$ is

$$
\psi\left(y\left(\xi_{0}\right), t\right)=\left[\begin{array}{ll}
1 & 0
\end{array}\right] \boldsymbol{e}^{A t} y\left(\xi_{0}\right)
$$

where

$$
A=\left[\begin{array}{cc}
\delta_{0} & 1 \\
0 & 0
\end{array}\right], \quad y\left(\xi_{0}\right)=\left[\begin{array}{c}
\phi\left(\xi_{0}\right) \\
\delta_{1}
\end{array}\right] .
$$

Proof: See the Appendix.

Observe that in contrast to [13, Lemma V.2] where the coefficients $\delta_{i}$ need to be positive, here $\delta_{i} \in \mathbb{R}$. This is because here, due to lack of knowledge on the derivative (or even on the differentiability) of the unknown signal $d(t)$, we consider only the first-order time derivative of $\phi$ (first-order comparison), whereas in [13], higher order derivatives of $\phi$ are considered (higher order comparison). For more information on the higher order comparison lemma, the reader is referred to Delimpaltadakis and Mazo [13] and the references therein.

Now, we employ Lemma VI.1, in order to construct an upper bound $\mu((x, w), t)$ of the triggering function $\tilde{\phi}(\xi(t ; x), w(t))$ that satisfies the conditions (15) (in the subspace $w>0$ ), which in turn implies that the zero-level sets of $\mu((x, w), t)$ are inner approximations of isochronous manifolds of DI (19) and satisfy the properties mentioned in Proposition IV.3. First, consider a compact connected set $Z \subset \mathbb{R}^{n}$ with $0 \in \operatorname{int}(Z)$, and the set $\mathrm{W}=[\underline{w}, \bar{w}]$, where $\bar{w}>\underline{w}>0$. Define the following sets:

$$
\begin{aligned}
& \Phi:=\bigcup_{x_{0} \in \mathrm{Z}}\left\{x \in \mathbb{R}^{n}: e=x_{0}-x, w \in \mathrm{W}, \tilde{\phi}((x, e, w)) \leq 0\right\} \\
& \mathrm{E}:=\left\{x_{0}-x \in \mathbb{R}^{n}: x_{0} \in \mathrm{Z}, x \in \Phi\right\} \\
& \Xi:=\Phi \times \mathrm{E} \times \mathrm{W} .
\end{aligned}
$$

For the remaining, we assume the following.

Assumption 2: The set $\Phi \subset \mathbb{R}^{n}$ is compact.

Assumption 2 is satisfied by most triggering functions $\phi(\cdot)$ in the literature (e.g., Lebesgue sampling and most cases of mixed triggering from Remark 2, the triggering functions of Tabuada [3] and Girard [5], etc.). Moreover, since $\Phi$ is assumed compact, then $\mathrm{E}$ is compact as well, which implies that $\Xi$ is compact.

Remark 3: As it is discussed after Theorem VI.2, the sets $\mathrm{Z}, \mathrm{W}, \Phi, \mathrm{E}, \Xi$ are constructed such that for all initial conditions $(x, 0, w) \in \mathrm{Z} \times \mathrm{E} \times \mathrm{W}$, the trajectories of DI (19) reach the boundary of $\Xi$ after (or at) the intersampling time $t=\tau((x, w))$. An alternative construction of such sets has been proposed in [7] and [13] and utilizes a given Lyapunov function for system (12) and its level sets.

The following theorem shows how the bound $\mu((x, w), t)$ is constructed.

Theorem VI.2: Consider the family of ETC systems (12), the DI (19) abstracting them, a homogenized triggering function $\tilde{\phi}(\xi(t ; x), w(t))$, the sets $\mathrm{Z}, \mathrm{W}, \Phi, \mathrm{E}, \Xi$ defined in (28), and let Assumptions 1 and 2 hold. Let $\delta_{0} \geq 0$ and $\delta_{1}>0$ be such that

$$
\begin{aligned}
& \forall(z, w, u) \in \Xi \times \Delta: \\
& \quad \frac{\partial \tilde{\phi}}{\partial z}(z, w) w^{\alpha+1} f_{e}\left(w^{-1} z, u\right) \leq \delta_{0} \tilde{\phi}(z, w)+\delta_{1}
\end{aligned}
$$

$$
\forall(z, w) \in \mathrm{Z} \times\{0\} \times \mathrm{W}: \delta_{0} \tilde{\phi}(z, w)+\delta_{1} \geq \varepsilon_{\delta}>0
$$

where $\varepsilon_{\delta}$ is an arbitrary positive constant. Let $r>\underline{w}$ be such that $D_{r}:=\left\{(x, w) \in \mathbb{R}^{n+1}:|(x, w)|=r, w \in \mathrm{W}\right\} \subset \mathrm{Z} \times \mathrm{W}$. For all $(x, w) \in \mathbb{R}^{n+1} \backslash\{0\}$ define the function

$$
\mu((x, w), t):=\left(\frac{|(x, w)|}{r}\right)^{\theta+1}\left[\begin{array}{ll}
1 & 0
\end{array}\right] e^{A\left(\frac{|(x, w)|}{r}\right)^{\alpha} t} y(x, w)
$$

where $A$ is as in (27) and

$$
y(x, w)=\left[\begin{array}{c}
\tilde{\phi}\left(\left(\frac{x}{|(x, w)|}, 0, r \frac{w}{|(x, w)|}\right)\right) \\
\delta_{1}
\end{array}\right] .
$$

The function $\mu((x, w), t)$ satisfies (15a), (15c), and (15d) for all $(x, w) \in\left(\mathbb{R}^{n} \times \mathbb{R}_{+}\right) \backslash\{0\}$, but condition (15b) is satisfied only in the cone

$$
\mathcal{C}=\left\{(x, w) \in \mathbb{R}^{n} \times \mathbb{R}_{+}:|x|^{2}+w^{2} \leq \frac{w^{2}}{\underline{w}^{2}} r^{2}\right\} \backslash\{0\}
$$

and $\forall t \in[0, \tau((x, w))]$.

Proof: See the Appendix.

Remark 4: Observe that under Assumptions 1 and 2, the term $\frac{\partial \tilde{\phi}}{\partial z}(z, w) w^{\alpha+1} f_{e}\left(w^{-1} z, u\right)$ is bounded for all $(z, w, u) \in$ $\Xi \times \Delta$, since $f_{e}$ is locally bounded, $\phi$ is continuously differentiable (implying that $\tilde{\phi}$ is also continuously differentiable for $w \neq 0), \tilde{\phi}(z, w)$ is bounded for all $(z, w) \in$ $\mathrm{Z} \times\{0\} \times \mathrm{W}$ and $\Xi \times \Delta$ is compact and does not contain any point $(z, 0, u)$. Thus, coefficients $\delta_{0} \geq 0$ and $\delta_{1}>$ 0 satisfying (29) always exist; e.g., $\delta_{0}=0$ and $\delta_{1}>$ $\max \left\{\epsilon_{\delta}, \sup _{(z, w, u) \in \Xi \times \Delta} \frac{\partial \tilde{\phi}}{\partial z}(z, w) w^{\alpha+1} f_{e}\left(w^{-1} z, u\right)\right\}$. In [13], a computational algorithm has been proposed, which computes the coefficients $\delta_{i}$ for a given ETC system and triggering function, by employing linear programming and satisfiabilitymodulo theory solvers (see, e.g., [23]).

Let us explain the intuition behind Theorem VI.2. First, observe that according to Lemma VI.1, the coefficients $\delta_{0}, \delta_{1}$ satisfying (29a), determine a function $\psi\left(y\left(\left(x, 0, w_{\star}\right)\right), t\right)$ that upper bounds $\sup \left\{\phi\left(\mathcal{X}_{t}^{\tilde{F}}\left(\left(x, 0, w_{\star}\right)\right)\right)\right\}$. The sets $\mathrm{Z}, \mathrm{W}, \Phi, \mathrm{E}, \Xi$ have been chosen such that the inequality

$$
\psi\left(y\left(\left(x, 0, w_{\star}\right)\right), t\right) \geq \sup \left\{\phi\left(\mathcal{X}_{t}^{\tilde{F}}\left(\left(x, 0, w_{\star}\right)\right)\right)\right\}
$$

holds for all $t \in\left[0, \tau\left(\left(x, w_{\star}\right)\right)\right]$. Now, introducing the scaling terms $\left(\frac{|(x, w)|}{r}\right)^{\alpha}, r \frac{x}{|(x, w)|}$, etc., which projects $\psi(\cdot)$ onto the spherical segment $D_{r}$ and transforms it into $\mu(\cdot)$, enforces that $\mu(\cdot)$ satisfies $(15 \mathrm{~b})$ and the scaling property $(15 \mathrm{c})$. Inequalities $\delta_{0} \geq 0, \delta_{1}>0$ and (29b) enforce that $\mu(\cdot)$ satisfies (15d). Finally, (15b) being satisfied only in the cone $\mathcal{C}$ stems from the fact that $0 \notin \operatorname{int}(W)$. Note that $W$ is chosen such that it is guaranteed that (29) is well-defined everywhere in $\Xi \times \Delta$.

The fact that $(15 b)$ is satisfied only in the cone $\mathcal{C}$ has the following implication.

Corollary VI.3. (See Theorem IV.4): Consider the family of ETC systems (12), the DI (19) abstracting them, a (homogenized) triggering function $\tilde{\phi}(\xi(t ; x), w(t))$, and let Assumptions 1 and 2 hold. Consider the function $\mu((x, w), t)$ from (30). 


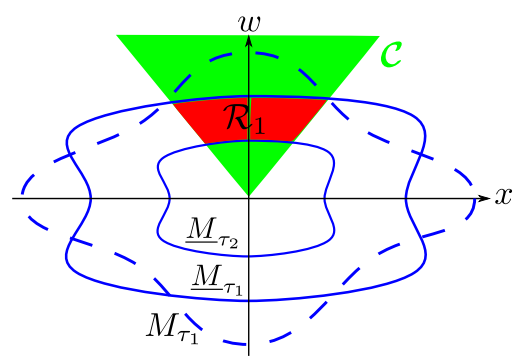

Fig. 3. Isochronous manifold $M_{\tau_{1}}$ (solid line) and approximations of isochronous manifolds $\underline{M}_{\tau_{1}}, \underline{M}_{\tau_{2}}$ (dashed lines). The set $\underline{M}_{\tau_{1}}$ inner approximates $M_{\tau_{1}}$ only inside the cone $\mathcal{C}$. The red region $\mathcal{R}_{1}$ contained between $\underline{M}_{\tau_{1}}$ and $\underline{M}_{\tau_{2}}$ and the cone $\mathcal{C}$ satisfies (11).

The sets $\underline{M}_{\tau_{\star}}=\left\{(x, w) \in \mathbb{R}^{n+1}: \mu\left((x, w), \tau_{\star}\right)=0\right\}$ innerapproximate isochronous manifolds $M_{\tau_{\star}}$ of DI (19) inside the cone $\mathcal{C}$, i.e., for all $(x, w) \in \underline{M}_{\tau_{\star}} \cap \mathcal{C}$

$$
\begin{aligned}
& \exists ! \kappa_{(x, w)} \geq 1 \text { s.t. } \kappa_{(x, w)}(x, w) \in M_{\tau_{\star}} \\
& \exists \lambda_{(x, w)} \in(0,1) \text { s.t. } \lambda_{(x, w)}(x, w) \in M_{\tau_{\star}} .
\end{aligned}
$$

Moreover, the sets $\underline{M}_{\tau_{\star}}$ satisfy the properties mentioned in Proposition IV.3.

Proof: It follows identical arguments to the proof of Theorem IV.4 in [13]. The only difference is that the arguments are now made for all $(x, w) \in \mathcal{C}$ and not for all $(x, w) \in \mathbb{R}^{n+1}$.

The implications of the aforementioned corollary are depicted in Fig. 3. According to Section IV-B, since the zero-level sets $\underline{M}_{\tau_{i}}$ of $\mu((x, w), t)$ inner-approximate isochronous manifolds inside $\mathcal{C}$, for the regions $\mathcal{R}_{i}$ that are delimited by consecutive approximations $\underline{M}_{\tau_{i}}$ and the cone $\mathcal{C}$ (see Fig. 3) it holds that: $\tau_{i} \leq \tau((x, w))$. Thus, given the set of times $\left\{\tau_{1}, \ldots, \tau_{q}\right\}$, the regions $\mathcal{R}_{i}$ are defined as the regions between consecutive approximations $\underline{M}_{\tau_{i}}$ and the cone $\mathcal{C}$

$$
\begin{aligned}
\mathcal{R}_{i}:= & \left\{(x, w) \in \mathcal{C}: \mu\left((x, w), \tau_{i}\right) \leq 0,\right. \\
& \left.\mu\left((x, w), \tau_{i+1}\right) \geq 0\right\} .
\end{aligned}
$$

As discussed in Section V-B, in a real-time implementation, given a measurement $x \in \mathbb{R}^{n}$, the controller checks to which region $\mathcal{R}_{i}$ the point $(x, 1) \in \mathbb{R}^{n+1}$ belongs, and correspondingly decides the next sampling time instant (see Fig. 2).

Remark 5: The innermost region $\mathcal{R}_{q}$ cannot be defined as in (32), as there is no $\tau_{q+1}$. For $\mathcal{R}_{q}$, it suffices that we write

$$
\mathcal{R}_{q}:=\left\{(x, w) \in \mathcal{C}: \mu\left((x, w), \tau_{q}\right) \leq 0\right\} .
$$

\section{B. Semiglobal Nature of Region-Based STC}

It is obvious that the regions $\mathcal{R}_{i}$ do not cover the whole $w=1$ hyperplane (which is where the state space of the original system is mapped), i.e., there exist states $x \in \mathbb{R}^{n}$ such that the point $(x, 1) \in \mathbb{R}^{n+1}$ does not belong to any region $\mathcal{R}_{i}$, and thus no STC intersampling time can be assigned to $x$. Let us demonstrate which set $\mathcal{B} \subseteq \mathbb{R}^{n}$ is covered by the partition created and show that it can be made arbitrarily large.
The set $\mathcal{B}$ is composed of all points $x \in \mathbb{R}^{n}$ such that $(x, 1)$ belongs to any region $\mathcal{R}_{i}$, i.e.,

$$
\mathcal{B}:=\left\{x \in \mathbb{R}^{n}:(x, 1) \in \bigcup_{i} \mathcal{R}_{i}\right\} .
$$

From the definition (32) of regions $\mathcal{R}_{i}$ and the scaling property (15c) of $\mu(\cdot)$, it follows that

$$
\bigcup_{i} \mathcal{R}_{i}=\mathcal{C} \cap\left\{(x, w) \in \mathbb{R}^{n} \times \mathbb{R}_{+}: \mu\left((x, w), \tau_{1}\right) \leq 0\right\} .
$$

By fixing $w=1$ in the expression (31) of $\mathcal{C}$ and in $\{(x, w) \in$ $\left.\mathbb{R}^{n} \times \mathbb{R}_{+}: \mu\left((x, w), \tau_{1}\right) \leq 0\right\}$, we get

$$
\begin{aligned}
& (x, 1) \in \mathcal{C} \Longleftrightarrow x \in\left\{x \in \mathbb{R}^{n}:|x|^{2} \leq \frac{r^{2}-\underline{w}^{2}}{\underline{w}^{2}}\right\}=: B_{1} \\
& (x, 1) \in\left\{(x, w) \in \mathbb{R}^{n} \times \mathbb{R}_{+}: \mu\left((x, w), \tau_{1}\right) \leq 0\right\} \Longleftrightarrow \\
& x \in\left\{x \in \mathbb{R}^{n}: \mu\left((x, 1), \tau_{1}\right) \leq 0\right\}=: B_{2} .
\end{aligned}
$$

Thus, we can write the set $\mathcal{B}$ as

$$
\mathcal{B}:=\left\{x \in \mathbb{R}^{n}: x \in B_{1}, x \in B_{2}\right\}=B_{1} \cap B_{2} .
$$

The set $B_{1}$ is depicted in Fig. 7 in the Appendix. Since $r>\underline{w}, B_{1}$ is nonempty. Moreover, we can choose $\underline{w}>0$ to be arbitrarily small without changing $r$, therefore we can make the set $B_{1}$ arbitrarily large. Finally, $B_{2}$ is nonempty (as it is the set delimited by $\underline{M}_{\tau_{1}}$ and $\mathcal{C}$ ) and, owing to the scaling property (15c) of $\mu(\cdot)$, it can be made arbitrarily large by selecting a sufficiently small $\tau_{1}$. Consequently, $\mathcal{B}$ is nonempty, and can be made arbitrarily large. Hence, region-based STC is applicable semiglobally in $\mathbb{R}^{n}$.

Remark 6: As discussed in [13], for fixed $\tau_{1}$ and $\tau_{q}$, as the total number $q$ of predefined times $\tau_{i}$ grows, the sets $\mathcal{R}_{i}$ become smaller (since the same set $\mathcal{B}$ is partitioned into more regions $\mathcal{R}_{i}$ ). This increases the accuracy of times $\tau_{i}$ as lower bounds of the actual ETC times $\tau(x)$, but also increases the online computational load of the controller, thus providing a tradeoff between performance and computations.

\section{NUMERICAL EXAMPLE}

Let us demonstrate how the proposed STC is applied to a perturbed uncertain system, and compare its performance to the STC of Liu and Jiang [4]. Consider the ETC system from [4]

$$
\dot{\zeta}_{1}=\zeta_{2}+g_{1}\left(\zeta_{1}, d_{1}\right), \quad \dot{\zeta}_{2}=u\left(\zeta, \varepsilon_{\zeta}\right)+g_{2}\left(\zeta_{2}\right)
$$

where $\left|g_{1}\left(\zeta_{1}, d_{1}\right)\right| \leq 0.1\left|\zeta_{1}\right|+0.1\left|d_{1}\right|$ and $\left|g_{2}\left(\zeta_{2}\right)\right| \leq 0.2\left|\zeta_{2}\right|^{2}$ are uncertain, and $d_{1}(t)$ is an unknown bounded disturbance with $\left|d_{1}(t)\right| \leq 4$. The ETC feedback $u$ is $u\left(\zeta, \varepsilon_{\zeta}\right)=$ $-\left(7.02\left|\zeta_{2}+\varepsilon_{\zeta_{2}}-p_{1}\right|-25.515\right)\left(\zeta_{2}+\varepsilon_{\zeta_{2}}-p_{1}\right)$, where $p_{1}=$ $-2.1\left(\zeta_{1}+\varepsilon_{\zeta_{1}}\right)$. The triggering function from Liu and Jiang [4], which is to be emulated, is

$$
\phi\left(\zeta, \varepsilon_{\zeta}\right)=\left|\varepsilon_{\zeta}(t)\right|^{2}-0.0049|\zeta(t)|^{2}-16
$$


which guarantees convergence to a ball (practical stability). First, we bring (36) to the form of (12), by writing

$$
\dot{\xi}(t)=\left[\begin{array}{c}
\dot{\zeta}_{1} \\
\dot{\zeta}_{2} \\
\dot{\varepsilon}_{\zeta_{1}} \\
\dot{\varepsilon}_{\zeta_{2}}
\end{array}\right]=\left[\begin{array}{c}
\zeta_{2}+0.1 d_{2} \zeta_{1}+0.1 d_{1} \\
u\left(\zeta, \varepsilon_{\zeta}\right)+0.2 d_{3} \zeta_{2}^{2} \\
-\zeta_{2}-0.1 d_{2} \zeta_{1}-0.1 d_{1} \\
-u\left(\zeta, \varepsilon_{\zeta}\right)-0.2 d_{3} \zeta_{2}^{2}
\end{array}\right]=f_{e}(\xi(t), d(t))
$$

where $d(t)=\left(d_{1}(t), d_{2}(t), d_{3}(t)\right) \in[-4,4] \times[-1,1]^{2}$, i.e., $\Delta=[-4,4] \times[-1,1]^{2}$. Observe that Assumption 1 is satisfied. Then, we construct the homogeneous DI abstracting (38) according to (19)

$$
\left(\begin{array}{c}
\dot{\xi}(t) \\
\dot{w}(t)
\end{array}\right)=\left[\begin{array}{c}
\left\{w^{2} f_{e}\left(w^{-1} \xi, d(t)\right): d(t) \in \Delta\right\} \\
0
\end{array}\right]
$$

and homogenize the triggering function as follows:

$$
\tilde{\phi}(\xi(t), w(t))=\left|\varepsilon_{\zeta}(t)\right|^{2}-0.0049|\zeta(t)|^{2}-16^{\sim} w^{2}(t) .
$$

The degree of homogeneity for both (39) and (40) is 1.

Next, we derive the $\delta_{i}$ coefficients according to Theorem VI.2 to determine the regions $\mathcal{R}_{i}$. We fix $\mathrm{Z}=[-0.1,0.1]^{2}$, $\mathrm{W}=\left[10^{-6}, 0.1\right]$ and define the sets $\Phi, \mathrm{E}, \Xi$ as in (28), where $\Phi$ is indeed compact. By employing the computational algorithm of Delimpaltadakis and Mazo [13], $\delta_{0} \approx 0.0353$ and $\delta_{1} \approx 0.3440$ are obtained. We choose $r=0.099$ such that $D_{r} \subset \mathrm{Z} \times \mathrm{W}$, and define $\mu((x, w), t)$ as in (30). Finally, the state space of DI (39) is partitioned into 434 regions $\mathcal{R}_{i}$ with $\tau_{1} \approx 63 \cdot 10^{-5}$ and $\tau_{i+1}=1.01 \tau_{i}$.

We ran a number of simulations to compare our approach to the approach of Liu and Jiang [4] and to the ideal performance of the emulated ETC (37). More specifically, we simulated the system for 100 different initial conditions uniformly distributed in a ball of radius 2 . The simulations' duration is 5s. As in [4], we fix: $g_{1}\left(\zeta_{1}, d_{1}\right)=0.1 \zeta_{1} \sin \left(\zeta_{1}\right)+0.1 d_{1}$, $d_{1}=4 \sin (2 \pi t)$, and $g_{2}\left(\zeta_{2}\right)=0.2 \zeta_{2}^{2} \sin \left(\zeta_{2}\right)$. The self-triggered sampler of Liu and Jiang [4] determines sampling times as follows: $t_{i+1}=t_{i}+\frac{1.54}{28\left(\left|x_{i}\right|+4\right)+29}$, where $x_{i}$ is the state measured at $t_{i}$. The total number of samplings for each simulation of all three schemes is depicted in Fig. 4. The average number of samplings per simulation was: 200.71 for region-based STC, 482.32 for STC [4], and 38.81 for ETC. We observe that region-based STC is in general less conservative than the STC of Liu and Jiang [4] while being more versatile as well. Recall that the main advantage of our approach is its versatility compared to the rest of the approaches, in terms of its ability to handle different performance specifications and different types of system's dynamics, provided that an appropriate triggering function is given. For example, the work in [4] is constrained to ISS systems, whereas our approach does not obey such a restriction. Finally, as expected, ETC leads to a smaller amount of samplings compared to both STC schemes.

We, also, present illustrative results for one particular simulation with initial condition $(-1,-1)$. Fig. 5 shows the trajectories of the system when controlled via region-based STC and the STC from Liu and Jiang [4], whereas Fig. 6 shows the time-evolution of intersampling times for the two schemes. Region-based STC

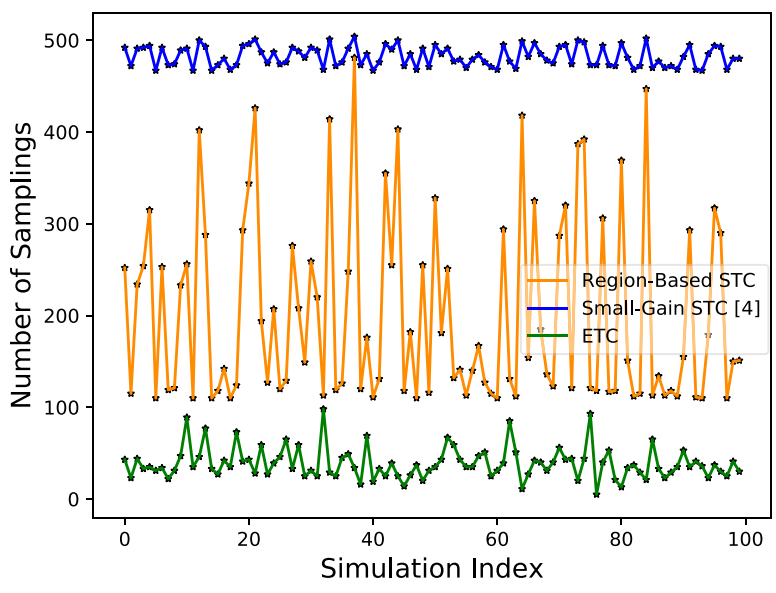

Fig. 4. Number of samplings for each simulation of region-based STC (orange), STC of Liu and Jiang [4] (blue), and ETC (37) (green).

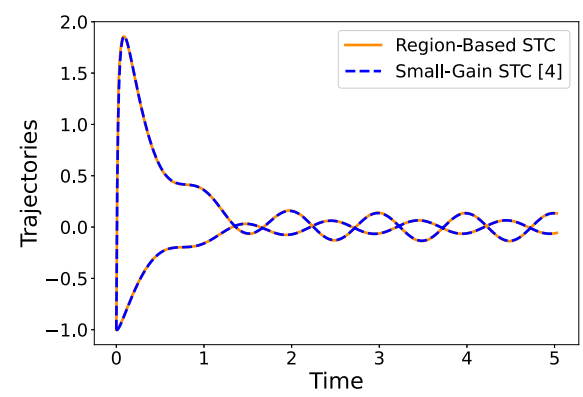

Fig. 5. Trajectories of system (36) with initial condition $(-1,-1)$, under region-based STC (orange lines) and the STC of Liu and Jiang [4] (dashed blue lines).

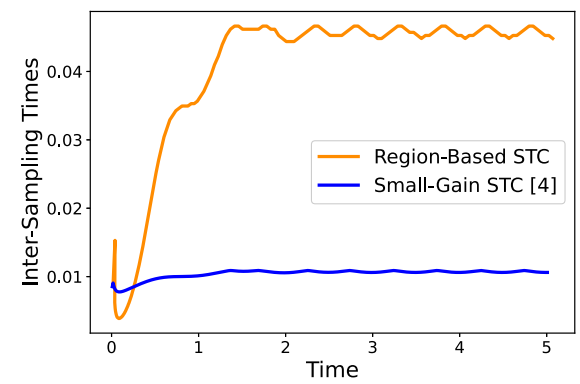

Fig. 6. Evolution of intersampling times during a simulation with initial condition $(-1,-1)$, for region-based STC (orange line) and the STC of Liu and Jiang [4] (blue line).

led to 166 samplings, whereas the STC of Liu and Jiang [4] led to 483 . We observe that while the performance of both schemes is the same (the trajectories are almost identical in Fig. 5), region-based STC leads to a smaller amount of samplings, i.e., less resource utilization. Moreover, from Fig. 6, we observe that, especially during the steady-state response, region-based STC performs considerably better in terms of sampling. However, there is a small period of time in the beginning of the simulation, when the trajectories overshoot far away from the origin and region-based STC gives faster sampling. Finally, we have to note 
that while we have not added more comparative simulations with the other STC schemes that address disturbances or uncertainties [10]-[12] for conciseness, simulation results have indicated that region-based STC is competitive to these approaches as well.

\section{CONCLUSION}

In this work, by extending the work of Delimpaltadakis and Mazo [13], we have proposed a region-based STC scheme for nonlinear systems with disturbances and uncertainties, which is able to provide different performance guarantees, depending on the triggering function that is chosen to be emulated. By employing a framework based on DIs and introducing ETC notions therein, we have extended significant results on ETC/STC to perturbed uncertain systems. Employing the renewed results, we have constructed approximations of isochronous manifolds of perturbed/uncertain systems, enabling region-based STC. The provided numerical simulations indicate that our approach while being more versatile is competitive with respect to other approaches as well, in terms of intersampling times. It is worth noting that region-based STC provides room for numerous extensions, due to the generic way of converting ETC to STC it offers. For example, (dynamic) output-feedback could easily be considered by incorporating the (controller's and) observer's dynamics into the system description. Hence, for future work, we will consider several extensions of the proposed STC (e.g., to systems with communication delays). Apart from that, we plan on utilizing the derived approximations of isochronous manifolds to construct timing models of perturbed uncertain nonlinear ETC systems for traffic scheduling in networks of ETC loops, building upon Delimpaltadakis and Mazo [19].

\section{APPENDIX}

Proof of Theorem V.2: According to the definition of intersampling times (18), for $\tau(\lambda(x, w))$, we have

$\tau(\lambda(x, w))=\inf \left\{t>0: \sup \left\{\tilde{\phi}\left(\mathcal{X}_{t}^{\tilde{F}}(\lambda(x, 0, w))\right)\right\} \geq 0\right\}$.

Employing the scaling property (4) and the fact that $\tilde{\phi}$ is homogeneous of degree $\theta$, we can write $\tau(\lambda(x, w))$ as

$$
\begin{aligned}
& \inf \left\{t>0: \sup \left\{\tilde{\phi}\left(\lambda \mathcal{X}_{\lambda^{\alpha} t}^{\tilde{F}}((x, 0, w))\right)\right\} \geq 0\right\} \\
& \quad=\inf \left\{t>0: \sup \left\{\lambda^{\theta+1} \tilde{\phi}\left(\mathcal{X}_{\lambda^{\alpha} t}^{\tilde{F}}((x, 0, w))\right)\right\} \geq 0\right\} \\
& \quad=\inf \left\{\lambda^{-\alpha} t>0: \sup \left\{\tilde{\phi}\left(\mathcal{X}_{t}^{\tilde{F}}((x, 0, w))\right)\right\} \geq 0\right\} \\
& =\lambda^{-\alpha} \tau((x, w)) .
\end{aligned}
$$

Proof of Lemma VI.1: Consider the restriction of ODE (22) to the set $\Xi$

$$
\dot{\xi}(t)=f(\xi(t), d(t)), \quad \xi(t) \in \Xi .
$$

Any solution of (41) is also a solution of (22) (possibly not a maximal one). Note that (24) is equivalent to

$$
\dot{\phi}\left(\xi\left(t ; \xi_{0}\right)\right) \leq \delta_{0} \phi\left(\xi\left(t ; \xi_{0}\right)\right)+\delta_{1}
$$

where $\xi\left(t ; \xi_{0}\right)$ is any solution of (41), with $\xi_{0} \in \Xi$. Observe that $\psi\left(y\left(\xi_{0}\right), t\right)$ is the solution to the scalar differential equation $\dot{\psi}=\delta_{0} \psi+\delta_{1}$ with initial condition $\psi_{0}=\phi\left(\xi_{0}\right)$

$$
\psi\left(y\left(\xi_{0}\right), t\right)=\left[\begin{array}{ll}
1 & 0
\end{array}\right] e^{A t} y\left(\xi_{0}\right)=e^{\delta_{0} t} \phi\left(\xi_{0}\right)+\frac{e^{\delta_{0} t}-1}{\delta_{0}} \delta_{1} .
$$

Thus, by employing the comparison lemma (see [22], pp. 102$103)$, from (42), we get that for any $d_{\star}(t)$ satisfying Assumption 1 and all $\xi_{0} \in \Xi$

$$
\phi\left(\xi\left(t ; \xi_{0}\right)\right) \leq \psi\left(y\left(\xi_{0}\right), t\right) \quad \forall t \in\left[0, t_{e, d_{\star}}\left(\xi_{0}\right)\right)
$$

where $\left[0, t_{e, d_{\star}}\left(\xi_{0}\right)\right)$ is the maximal interval of existence of solution $\xi\left(t ; \xi_{0}\right)$ to ODE (41) under the realization $d(t)=d_{\star}(t)$. The time $t_{e, d_{\star}}\left(\xi_{0}\right)$ is defined as the time when $\xi\left(t ; \xi_{0}\right)$, under the realization $d(t)=d_{\star}(t)$, leaves the set $\Xi$

$t_{e, d_{\star}}\left(\xi_{0}\right)=\sup \left\{\tau>0: d(t)=d_{\star}(t), \xi\left(t ; \xi_{0}\right) \in \Xi \forall t \in[0, \tau)\right\}$.

Since (43) holds for all $d_{\star}(t)$ satisfying Assumption 1, we can conclude that $\psi\left(y\left(\xi_{0}\right), t\right)$ bounds all solutions of DI (23) starting from $\xi_{0} \in \Xi$ as follows:

$$
\sup \left\{\phi\left(\mathcal{X}_{t}^{F}\left(\xi_{0}\right)\right)\right\} \leq \psi\left(y\left(\xi_{0}\right), t\right) \quad \forall t \in\left[0, \inf _{d_{\star}} t_{e, d_{\star}}\left(\xi_{0}\right)\right) .
$$

Finally, note that $\inf _{d_{\star}} t_{e, d_{\star}}\left(\xi_{0}\right)$ represents the smallest possible $\Xi$-escape time among all trajectories generated by DI (23), i.e., $\inf _{d_{\star}} t_{e, d_{\star}}\left(\xi_{0}\right)=\inf \left\{t>0: \mathcal{X}_{t}^{F}\left(\xi_{0}\right) \nsubseteq \Xi\right\}=$ $t_{e}\left(\xi_{0}\right)$. Hence, we can conclude that

$$
\sup \left\{\phi\left(\mathcal{X}_{t}^{F}\left(\xi_{0}\right)\right)\right\} \leq \psi\left(y\left(\xi_{0}\right), t\right) \quad \forall t \in\left[0, t_{e}\left(\xi_{0}\right)\right) .
$$

Proof of Theorem VI.2: First notice that under item 4) of Assumption 1, (15a) holds:

$\mu((x, w), 0)=\left(\frac{|(x, w)|}{r}\right)^{\theta+1} \tilde{\phi}\left(\left(r \frac{x}{|(x, w)|}, 0, r \frac{w}{|(x, w)|}\right)\right)<0$

for all $(x, w) \in \mathbb{R}^{n+1} \backslash\{0\}$. Moreover, observe that $\mu(\cdot, \cdot)$ satisfies the time-scaling property $(15 \mathrm{c})$ by construction. It remains to prove that $\mu(\cdot, \cdot)$ satisfies $(15 \mathrm{~b})$ and $(15 \mathrm{~d})$.

In order to prove that $\mu(\cdot, \cdot)$ satisfies $(15 \mathrm{~b})$, as already explained in Section VI-A, we follow the following steps.

1) We show that the coefficients $\delta_{0}, \delta_{1}$ satisfying (29a) determine a function $\psi\left(y\left(\left(x, 0, w_{\star}\right)\right), t\right)$ satisfying (44).

2) Using the sets $\mathrm{Z}, \mathrm{W}, \mathrm{E}, \Phi, \Xi$, we show that $\psi\left(y\left(\left(x, 0, w_{\star}\right)\right), t\right)$ satisfies $(45)$.

3) Finally, observing that $\mu$ is obtained by a projection of $\psi$ to $D_{r}$, we show that $\mu$ satisfies (15b) [see (49)].

Let us formally prove it. Assumption 1 implies that $\tilde{F}(\xi, w) \subseteq \mathbb{R}^{2 n+1}$ is nonempty, compact, and convex for any $(\xi, w) \in \mathbb{R}^{2 n+1} \backslash\{0\}$ and outer semicontinuous. These conditions ensure existence and extendability of solutions for each initial condition [21]. According to Lemma VI.1 and since $\Xi$ is compact, the coefficients $\delta_{0}, \delta_{1}$ satisfying (29a), determine a function $\psi\left(y\left(\left(x, e, w_{\star}\right)\right), t\right)$ such that for all $(x, e, w)$ 


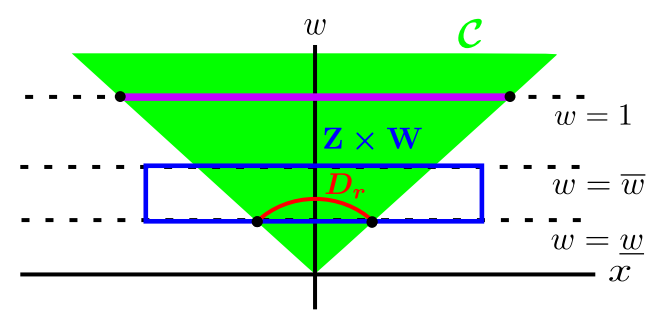

Fig. 7. Sets $\mathrm{Z} \times \mathrm{W}$ (region contained in blue box), $D_{r}$ (red spherical segment), and the cone $\mathcal{C}$ (green) from (31). The subset of the hyperplane $w=1$ painted in purple represents the set $B_{1}$ from (33).

$\in \Xi: \psi(y((x, e, w)), t) \geq \sup \left\{\phi\left(\mathcal{X}_{t}^{\tilde{F}}((x, e, w))\right)\right\} \quad \forall t \in[0$ $\left.t_{e}((x, e, w))\right]$, where $t_{e}((x, e, w))$ is defined in (25) as the time when $\mathcal{X}_{t}^{\tilde{F}}((x, e, w))$ leaves the set $\Xi$. Since we are only interested in initial conditions with the measurement error component being 0 , we write

$$
\begin{aligned}
& \psi(y((x, 0, w)), t) \geq \sup \left\{\phi\left(\mathcal{X}_{t}^{\tilde{F}}((x, 0, w))\right)\right\} \\
& \forall(x, 0, w) \in \Xi \text { and } \forall t \in\left[0, t_{e}((x, 0, w))\right] .
\end{aligned}
$$

Observe that for all initial conditions $(x, 0, w) \in \mathrm{Z} \times \mathrm{E} \times \mathrm{W}$, the sets $\Phi$ and $\mathrm{E}$ are exactly such that $\xi(t ;(x, 0)) \notin \Phi \times \mathrm{E} \Rightarrow$ $\phi(\xi(t ;(x, 0)))>0$, where $\xi(\cdot)$ represents the $\xi$-component of solutions of DI (19) (since $w(t)$ remains constant along solutions of DI (19), we neglect it). Thus, all trajectories that start from any initial condition $(x, 0, w) \in \mathrm{Z} \times \mathrm{E} \times \mathrm{W}$ reach the boundary of $\Xi=\Phi \times \mathrm{E} \times \mathrm{W}$ after (or at) the intersampling time $\tau((x, w))$, i.e., $\tau((x, w)) \leq t_{e}((x, e, w))$ for all $(x, w) \in \mathrm{Z} \times \mathrm{W}$. Thus, employing (44), we write

$$
\begin{aligned}
& \psi(y((x, 0, w)), t) \geq \sup \left\{\phi\left(\mathcal{X}_{t}^{\tilde{F}}((x, 0, w))\right)\right\} \\
& \forall(x, w) \in \mathrm{Z} \times \mathrm{W} \text { and } \forall t \in[0, \tau((x, w))] .
\end{aligned}
$$

Now, consider any point $\left(x_{0}, w_{0}\right) \in D_{r} \subseteq \mathrm{Z} \times \mathrm{W}$. Observe that $\mu\left(\left(x_{0}, w_{0}\right), t\right)=\psi\left(y\left(\left(x_{0}, 0, w_{0}\right)\right), t\right)$. Thus, since $D_{r} \subseteq$ $\mathrm{Z} \times \mathrm{W}$, from (45), we get

$$
\begin{aligned}
\mu\left(\left(x_{0}, w_{0}\right), t\right) & \geq \sup \left\{\phi\left(\mathcal{X}_{t}^{\tilde{F}}\left(\left(x_{0}, 0, w_{0}\right)\right)\right)\right\} \\
& \forall\left(x_{0}, w_{0}\right) \in D_{r} \text { and } \forall t \in\left[0, \tau\left(\left(x_{0}, w_{0}\right)\right)\right]
\end{aligned}
$$

To prove that $\mu(\cdot)$ satisfies (15b) in the cone $\mathcal{C}$ from (31), we have to show that $(46)$ holds for all $(x, w) \in \mathcal{C}$. First, observe that $\mathcal{C}$ is defined as the cone stemming from the origin with its extreme vertices being all points in the intersection $D_{r} \cap \mathrm{Z} \times \mathrm{W}$ (see Fig. 7). Thus, since $D_{r}$ is a spherical segment, for any point $(x, w) \in \mathcal{C}$ there always exists a $\lambda>0$ and a point $\left(x_{0}, w_{0}\right) \in$ $D_{r}$ such that $(x, w)=\lambda\left(x_{0}, w_{0}\right)$. If we interchange $\left(x_{0}, w_{0}\right)$ with $\lambda^{-1}(x, w)$ in (46), we get

$$
\begin{aligned}
& \mu\left(\lambda^{-1}(x, w), t\right) \geq \sup \left\{\phi\left(\mathcal{X}_{t}^{\tilde{F}}\left(\lambda^{-1}(x, 0, w)\right)\right)\right\} \\
& \forall(x, w) \in \mathcal{C} \text { and } \forall t \in\left[0, \tau\left(\lambda^{-1}(x, w)\right)\right] .
\end{aligned}
$$

But, from (4), (15c), and Theorem (V.2), we get

$$
\begin{aligned}
\bullet \sup & \left\{\phi\left(\mathcal{X}_{t}^{\tilde{F}}\left(\lambda^{-1}(x, 0, w)\right)\right)\right\} \\
= & \lambda^{-\theta-1} \sup \left\{\phi\left(\mathcal{X}_{\lambda^{-\alpha} t}^{\tilde{F}}((x, 0, w))\right)\right\} \\
& \bullet \mu\left(\lambda^{-1}(x, w), t\right)=\lambda^{-\theta-1} \mu\left((x, w), \lambda^{-\alpha} t\right) \\
& \bullet \tau\left(\lambda^{-1}(x, w)\right)=\lambda^{\alpha} \tau((x, w)) .
\end{aligned}
$$

Incorporating (48) into (47), we finally get

$$
\begin{aligned}
& \mu((x, w), t) \geq \sup \left\{\phi\left(\mathcal{X}_{t}^{\tilde{F}}((x, 0, w))\right)\right\} \\
& \forall(x, w) \in \mathcal{C} \text { and } \forall t \in[0, \tau((x, w))]
\end{aligned}
$$

i.e., $\mu(\cdot)$ satisfies $(15 b)$ in $\mathcal{C}$.

Finally, let us prove that $\mu(\cdot)$ satisfies $(15 \mathrm{~d})$. Observe that since $\delta_{0} \geq 0, \delta_{1}>0$, and (29b) holds, then $\mu((x, w), t)$ and $\dot{\mu}((x, w), t)$ are strictly increasing w.r.t. $t$ (for a more detailed proof, see [13]). Thus, since $\mu((x, w), 0)<0$, then for any $(x, w) \in \mathbb{R}^{n+1} \backslash\{0\}, \exists ! \tau^{\downarrow}(x, w)>0$ such that $\mu\left((x, w), \tau^{\downarrow}((x, w))\right)=0$.

\section{REFERENCES}

[1] K.-E. Åarzén, “A simple event-based PID controller," IFAC Proc. Vol., vol. 32, no. 2, pp. 8687-8692, 1999.

[2] K. J. Astrom and B. M. Bernhardsson, "Comparison of Riemann and Lebesgue sampling for first order stochastic systems," in Proc. 41st IEEE Conf. Decis. Control, 2002, vol. 2, pp. 2011-2016.

[3] P. Tabuada, "Event-triggered real-time scheduling of stabilizing control tasks," IEEE Trans. Autom. Control, vol. 52, no. 9, pp. 1680-1685, Sep. 2007.

[4] T. Liu and Z.-P. Jiang, "A small-gain approach to robust event-triggered control of nonlinear systems," IEEE Trans. Autom. Control, vol. 60, no. 8, pp. 2072-2085, Aug. 2015.

[5] A. Girard, "Dynamic triggering mechanisms for event-triggered control," IEEE Trans. Autom. Control, vol. 60, no. 7, pp. 1992-1997, Jul. 2015.

[6] M. Velasco, J. Fuertes, and P. Marti, "The self triggered task model for realtime control systems," in Proc. Work-In-Prog. Session 24th IEEE RealTime Syst. Symp., 2003, pp. 67-70, doi: 10.1109/TCYB.2020.2980048.

[7] A. Anta and P. Tabuada, "To sample or not to sample: Self-triggered control for nonlinear systems," IEEE Trans. Autom. Control, vol. 55, no. 9 , pp. 2030-2042, Sep. 2010.

[8] M. Mazo Jr., A. Anta, and P. Tabuada, "An ISS self-triggered implementation of linear controllers," Automatica, vol. 46, no. 8, pp. 1310-1314, 2010.

[9] X. Wang and M. D. Lemmon, "Self-triggering under state-independent disturbances," IEEE Trans. Autom. Control, vol. 55, no. 6, pp. 1494-1500, Jun. 2010.

[10] M. D. Di Benedetto, S. Di Gennaro, and A. D'innocenzo, "Digital selftriggered robust control of nonlinear systems," Int. J. Control, vol. 86, no. 9, pp. 1664-1672, 2013.

[11] U. Tiberi and K. H. Johansson, "A simple self-triggered sampler for perturbed nonlinear systems," Nonlinear Anal.: Hybrid Syst., vol. 10, no. 1, pp. 126-140, 2013.

[12] D. Tolic, R. G. Sanfelice, and R. Fierro, "Self-triggering in nonlinear systems: A small gain theorem approach," in Proc. Mediterranean Conf. Control Autom., 2012, pp. 941-947.

[13] G. Delimpaltadakis and M. Mazo, "Isochronous partitions for region-based self-triggered control," IEEE Trans. Autom. Control, to be published, doi: 10.1109/TAC.2020.2994020.

[14] W. P. M. H. Heemels, K. H. Johansson, and P. Tabuada, "An introduction to event-triggered and self-triggered control," in Proc. IEEE Conf. Decis. Control, 2012, pp. 3270-3285.

[15] K. Hashimoto, Y. Yoshimura, and T. Ushio, "Learning self-triggered controllers with Gaussian processes," IEEE Trans. Cybern., to be published, doi: 10.1109/TCYB.2020.2980048. 
[16] M. J. Khojasteh, V. Dhiman, M. Franceschetti, and N. Atanasov, "Probabilistic safety constraints for learned high relative degree system dynamics," in Proc. 2nd Conf. Learn. Dyn. Control, 2020, pp. 781-792.

[17] E. Bernuau, D. Efimov, W. Perruquetti, and A. Polyakov, "On an extension of homogeneity notion for differential inclusions," in Proc. Eur. Control Conf., 2013, pp. 2204-2209.

[18] A. Anta and P. Tabuada, "Exploiting isochrony in self-triggered control," IEEE Trans. Autom. Control, vol. 57, no. 4, pp. 950-962, Apr. 2012.

[19] G. Delimpaltadakis and M. Mazo, "Traffic abstractions of nonlinear homogeneous event-triggered control systems," in Proc. IEEE 59th Conf. Decis. Control, 2020, pp. 4991-4998.

[20] M. Kawski, "Geometric homogeneity and stabilization," in Nonlinear Control Systems Design. Amsterdam, The Netherlands: Elsevier, 1995, pp. $147-152$.

[21] A. F. Filippov, Differential Equations With Discontinuous Righthand Sides. Norwell, MA, USA: Kluwer, 1988.

[22] H. K. Khalil, Nonlinear Systems, vol. 2. Englewood Cliffs, NJ, USA Prentice-Hall, 1996, no. 5, pp. 5-1.

[23] S. Gao, S. Kong, and E. M. Clarke, "dReal: An SMT solver for nonlinear theories over the reals," in Proc. Int. Conf. Autom. Deduction, 2013, pp. 208-214.

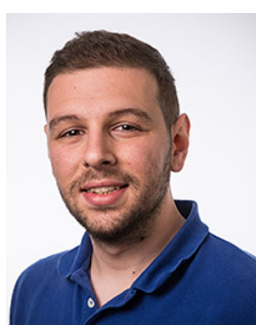

Giannis Delimpaltadakis (Student Member, IEEE) received the Diploma (joint B.Sc. and M.Sc. degrees) in electrical and computer engineering from the National Technical University of Athens, Athens, Greece, in 2017. $\mathrm{He}$ is currently working toward the Ph.D. degree with the Delft Center for Systems and Control, Delft University of Technology, Delft, The Netherlands.

His research interests include eventtriggered and self-triggered control, nonlinear systems, and networked control systems.

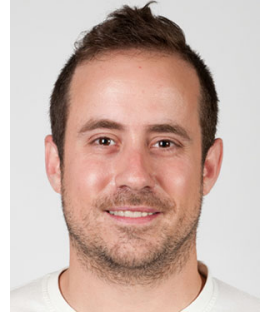

Manuel Mazo, Jr. (Senior Member, IEEE) received the Telecommunications Engineering "Ingeniero" degree from the Polytechnic University of Madrid, Madrid, Spain, in 2003, the "Civilingenjör" degree in electrical engineering from the Royal Institute of Technology, Stockholm, Sweden, in 2003, and the M.Sc. and Ph.D. degrees in electrical engineering from the University of California, Los Angeles, Los Angeles, CA, USA, in 2007 and 2010, respectively.

$\mathrm{He}$ is currently an Associate Professor with the Delft Center for Systems and Control, Delft University of Technology, Delft, The Netherlands. Between 2010 and 2012, he held a joint Postdoctoral position at the University of Groningen, Groningen, and the innovation centre INCAS3, The Netherlands. His main research interest includes the formal study of problems emerging in modern control system implementations and, in particular, the study of networked control systems and the application of formal verification and synthesis techniques to control.

Dr. Mazo, Jr. was the recipient of a University of Newcastle Research Fellowship (2005), the Spanish Ministry of Education/UCLA Fellowship (2005-2009), the Henry Samueli Scholarship from the UCLA School of Engineering and Applied Sciences (2007/2008), and an ERC Starting Grant (2017). 